\title{
Evolutionary Game Analysis of Competitive Information Dissemination on Social Networks: An Agent-Based Computational Approach
}

\author{
Qing Sun ${ }^{1,2}$ and Zhong Yao ${ }^{1}$ \\ ${ }^{1}$ School of Economics and Management, Beihang University, Beijing 100191, China \\ ${ }^{2}$ School of Computer Science and Engineering, Beihang University, Beijing 100191, China \\ Correspondence should be addressed to Zhong Yao; iszhyao@buaa.edu.cn
}

Received 22 August 2014; Accepted 5 December 2014

Academic Editor: L. W. Zhang

Copyright ( 92015 Q. Sun and Z. Yao. This is an open access article distributed under the Creative Commons Attribution License, which permits unrestricted use, distribution, and reproduction in any medium, provided the original work is properly cited.

Social networks are formed by individuals, in which personalities, utility functions, and interaction rules are made as close to reality as possible. Taking the competitive product-related information as a case, we proposed a game-theoretic model for competitive information dissemination in social networks. The model is presented to explain how human factors impact competitive information dissemination which is described as the dynamic of a coordination game and players' payoff is defined by a utility function. Then we design a computational system that integrates the agent, the evolutionary game, and the social network. The approach can help to visualize the evolution of $\%$ of competitive information adoption and diffusion, grasp the dynamic evolution features in information adoption game over time, and explore microlevel interactions among users in different network structure under various scenarios. We discuss several scenarios to analyze the influence of several factors on the dissemination of competitive information, ranging from personality of individuals to structure of networks.

\section{Introduction and Prior Work}

The emergent and rapid development of online social networking applications has changed the way in which both consumers and enterprises interact and collaborate with each other. Online social networking applications provide the engaged individuals with collaborative environment to share information or ideas with their neighbors, where the total effect is greater than the sum of individual effects. In social commerce, the dissemination of product-related information is affected by individuals' actions, which are strongly determined by their characteristics and often influenced by the decisions of other individuals. This kind of information diffusion on the social network, including social advertisements, word-of-mouth, and comments, finally influences the buying behavior of the potential consumers. The features and patterns of competitive information dissemination will affect the product-related information spreading, such as competitive advertisement and positive or negative word-ofmouth because of their commercial feature. Therefore, it is important to study how to model and analyze the competitive diffusion through social network.

Epidemic models have been widely adopted by researchers for information dissemination due to the analogy between epidemics and the spread of information. The underlying assumption of these models is that individuals adopt a new behavior with a probability when they interact with others who have already adopted it [1]. Gruhl et al. [2] investigate the adoption of the classic Susceptible-InfectedRemoved (SIR) model for information dissemination. Yang and Leskovec [3] developed a linear influence model to focus on influence of individual node on the rate of dissemination through the implicit network. Lü et al. [4] propose a modified SIR model to describe the information diffusion in the small-world network, proposing three different spreading rules from the standard SIR model: memory effects, social reinforcement, and nonredundancy of contacts, in which the influence of social network structure is considered and analyzed. These studies have macroscopically committed 
to the description of information diffusion through social networks.

In recent years, researchers gradually observe that game behaviors between individuals in social network, being the foundation of the social group behavior, raise social diffusions, which were always discussed in the microlevel in prior related research. Micronodes in network constantly adjust their strategy according to the behavior of other nodes, through the dynamic game with other nodes to maximize their own interests, and all the behaviours of individual node finally form the social network group behavior. Therefore, game-theoretic models, as a new perspective of interpreting social diffusion, are increasingly adopted by computer scientists for analyzing network behaviors. Game theory is a set of analytical tools and solution concepts, which provides explanatory and predicting power in interactive decision situations, when the aims, goals, and preferences of the participating players are potentially in conflict [5]. Unlike the classic game theory, which assumes that gaming exists between two individuals and happens only one-off, the evolutionary game theory has opened up related hypothesis limitations. It introduces the concept of time and space, considering the game as the summary of historical experience and individual learning, which makes it advantageous for social network study. Several papers have applied evolutionary game theory method to explain social diffusion. Kostka et al. [6] examined the dissemination of competing rumors in social network, using concepts of game theory and location theory, modelling the selection of starting nodes for the rumors as a strategy game. Meier et al. [7] presented a virus propagation game model, finding that the Windfall of Friendship does not increase monotonically with stronger relationships. Zinoviev et al. $[8,9]$ adopted game theoretic models to understand human aspects of information dissemination in which personalities of individuals are considered. Jiang et al. [10] examined the evolutionary process of knowledge sharing among users in social network and designed a computational experimental system, developing a mixed learning algorithm based on individual's historical game strategy, neighbors' strategy, and information noise.

Applying evolutionary game theory in social diffusion study presumes that the game process between individuals is not only determined by a single rival or individual, but also all individuals in the neighborhood. Meanwhile, it takes the historical game experience for future game behavior into consideration. The benefit of each individual is evaluated by the accumulated result of gaming many times. Individuals benefit from the game with its neighbors; at the same time, the benefit can be observed with comparisons to adjust their game strategies, trying to achieve optimum benefits and reach the overall game equilibrium. Evolutionary game theory also has advantages on competitive social behaviors and social diffusions. Alon et al. [11] introduced a gametheoretic model of competitive dissemination of technologies, advertisements, or influence through a social network. Wang et al. [12] proposed the stochastic game net model for analyzing competitive network behaviors. Takehara et al. [13] introduced and studied a deterministic model for competitive information diffusion on social networks. In contrast to many other game theoretic models for the diffusion of information and innovation $[14,15]$, the model considered competition between different innovations spreading instead of discussing a single one.

This paper is an extension of recent works, including the method suggested by Jiang et al. [16] and the results described by Yu et al. [17]. The dissemination of information is modeled as the dynamic of coordination game, in which player's payoff is defined by a utility function and several cases are analyzed to reach the conclusion that the spreading rate is influenced by characteristics of individuals and several other factors. Different from the models of competitive information diffusion introduced in prior work, we adopt a framework for describing competitive information dissemination based on a game-theoretic model and multiagent-based dynamics. In this framework, we target several similar product-related pieces of information that competes with each other. Consumers may participate in discussing, adopting, and spreading one of them. Totally different from news or opinions dissemination that is always described as the spreading of the virus, consumers always make product-related decision for the utility motivation. In general, the utility is dynamically determined by factors from the environment or from his personal reasons. Meanwhile, prior research has shown that different network structures topology has great impact on social commerce. Therefore, both sociological and psychological characteristics are explicitly considered in our model as the novelty. In this model, information passing intrinsically involves both sides considering their characteristics: selfperceived knowledge, brand loyalty, and social conformity, which further determine their decisions of whether or not to forward the information. The decisions are also based on the global properties of the network, such as the knowledge dynamic all through the network. These factors finally bring different results of disseminations of different information that compete with each other. Based on the dynamic game model and strategy updating rules, we analyze competitive information propagation and the affecting factors.

The remainder of this paper is organized as follows. Section 2 presents the overview of competitive dissemination model based on evolutionary game theory, social network, and multiagent theory and introduces the individual's utility function. Section 3 discusses the dynamics and updating rules for the model we build, proposes the assumptions, and explains the model. In Section 4 we use an agent-based computational approach for simulation.

\section{Problem Statement}

We propose a different, global point of view regarding the incentives that govern the diffusion process. Suppose we have several firms that would like to advertise competitive products via "viral marketing." Each firm initially targets a small subset of users, in the hope that the social advertisements about their product would spread throughout the network. However, a user that participates in discussing and spreading product-related information is reluctant to participate in another one. 
TABLE 1: Game payoff matrix.

\section{Player $i$}

$a$

\begin{tabular}{lcc} 
& $a$ & $b$ \\
\hline Player $j$ & & \\
$a$ & $U_{a a}, U_{a a}$ & $U_{a b}, U_{b a}$ \\
$b$ & $U_{b a}, U_{a b}$ & $U_{b b}, U_{b b}$ \\
\hline
\end{tabular}

For simplicity, we consider two pieces of information competing over the social network, represented by information $A$ or $B$. They are about the same kind of product with different brands (e.g., Smartphones of iPhone or Samsung). Which information is adopted by the individual is determined by his self-perceived knowledge and herd mentality. Information dissemination is caused by individuals' interaction with his neighbours for sharing knowledge and opinion. This interaction, which arouses changes of individuals' acceptance and preference, could be considered as a game based on information exchange. Individuals update their strategies according to their payoff and the influence of their neighbours. The change of individual's information level for the product affects the individual's preference and accordingly affects his choice of consumption.

2.1. Evolutionary Game Model. In the classic game-theoretic model, the consumers are defined as players and each player has only one state. We take each node in online social network as players in game. The game is played in period $t=\{n, n=$ $1,2,3, \ldots\}$ in online social networks that could be described as an undirected graph.

First of all, we assume that all players engaged in the evolutionary game are pure strategists, and each player could choose only one of the strategies; strategy $a$ is participating in discussion and spreading of information for information $A$ (corporation) and strategy $b$ is participating in discussion and spreading of information $B$ (defection). Then the set of available strategies for node $i$ could be described as twodimensional vectors:

$$
S_{i}=\left(\begin{array}{l}
1 \\
0
\end{array}\right) \text { or }\left(\begin{array}{l}
0 \\
1
\end{array}\right),
$$

where $S_{i}$ is the strategy of node $i$. In each round, the node games with all of its neighbours (friends) and profit. The payoff matrix $M$ is defined by the utility function $U(i)$ illustrated in Table 1.

In Table $1, U_{i j}, i, j \in(a, b)$, represents the utility for the player according to what strategy he chooses. The evolutionary game equilibrium can be detected by the dynamic replication method $[5,18]$ that can provide theoretical support for macroscopic decisions on information dissemination, ignoring network structure and environmental factors. Then, the expected cooperation (defection) benefit from knowledge sharing can be expressed as

$$
\begin{aligned}
& U_{i}(C)=p * U_{a a}+(1-p) * U_{a b}, \\
& U_{i}(D)=p * U_{b a}+(1-p) * U_{b b},
\end{aligned}
$$

where $p$ is the percentage of nodes holding a cooperative attitude (strategy $a) \cdot U_{i}(C)$ and $U_{i}(D)$, respectively, stand for the benefit that node $i$ get in current round of game.

From the dynamic replication, the average benefit of the whole group is

$$
\bar{U}=p * U_{i}(C)+(1-p) U_{i}(D) .
$$

The state of the evolutionary system over time for different initial value of $p$ can be evaluated by the diffusion rate $F(p)$ :

$$
\begin{aligned}
& F(p) \\
& =\frac{d p}{d t}=p\left(U_{i}(C)-\bar{U}\right) \\
& =p(1-p)\left[\left(U_{a a}-U_{a b}-U_{b a}+U_{b b}\right) * p+\left(U_{a b}-U_{b b}\right)\right] .
\end{aligned}
$$

Therefore, the game has three possible solutions to reach equilibrium:

$$
\begin{gathered}
p=0,1, \frac{U_{a b}-U_{b b}}{U_{a a}-U_{a b}-U_{b a}+U_{b b}}, \\
p^{*}=\frac{U_{a b}-U_{b b}}{U_{a a}-U_{a b}-U_{b a}+U_{b b}},
\end{gathered}
$$

where $p^{*}$ is the value with which the group evolutionary will get the game equilibrium. This method can get the evolutionary equilibrium independent of environmental factors and network structure. However, the competitive dissemination we discuss in this paper is more complex. The historical game path, the impacts of environment, and the interactive users' decision should be taken into consideration comprehensively. In this context, the equilibrium cannot be simply and solely determined by the traditional dynamic replication method. In this paper, we choose the method of computational simulation approach so as to get a more reasonable explanation and description for competitive information dissemination over the social networks.

2.2. Utility Definition. The impact of other individuals' behavior has been extensively studied in social psychology and marketing. In general, many early past findings (Asch [19] and Schachter [20]) suggest that individuals have a tendency to behave in accordance with group or social norms and behave negatively toward opinions that deviate from these norms. Based upon this premise, Deutsch and Gerard [21] further developed the distinction between normative social influence (pressure to conform to the expectations of others) and informational influence (individual's acceptance of persuasive argument(s) of others). Meanwhile, other researches [22, 23] (McQuail [22] and Flanagin and Metzger [23]) proved that people expect two types of value to engage in community: information value and social support. We refer to the conclusion and define the utility function when game continues as follows.

(1) Self-Perceived Knowledge Model. People feel that the message they spread can help others make informed decision 
of purchase. Product-related information is now being overwhelmed from a constantly increasing amount of advertising. Meanwhile, it can also be obtained by interpersonal sources (Feick and Price [24]). Self-perceived knowledge level helps to reduce the risk when making adopting and purchasing decision. In this paper, we define $k_{i} \in[0,1](i=A$ or $B)$ as the quantitative measure of self-perceived knowledge for the product of brand $i$. This kind of knowledge can either be gained from mass media or word-of-mouth prevalent in the social network. The quantity of 1 represents full knowledge of the product, which means the player knows everything about the background of the product. The quantity of 0 represents no prior knowledge of the product, which means the product is totally new to the player. A value of $k \in(0,1)$ represents partial background knowledge of the product, which means the player knows something about the product (such as "I know something about the Smartphone of this brand"). We describe the self-perceived knowledge level for both the two brands of play $i$ as a vector $K_{i}=\left(\begin{array}{l}k_{A} \\ k_{B}\end{array}\right)$. The consistent conclusion can easily be reached in the real social commerce circumstance, because the more self-perceived knowledge a consumer has, the lower risk and cost he will pay for consumptions.

Moreover, we observe that knowledge will transfer within players as game continues; under some situations even new knowledge will be created. In order to describe the knowledge dynamics during the game process, we assume that there are two independent kinds of knowledge, each kind corresponding to one of the competitive pieces of information of product. For example, $k_{i}$ and $k_{j}$ are referring to different products. They interact as the following rules.

(1) Interacting with a player having different kinds of knowledge does not increase knowledge; $k_{i}$ and $k_{j}$ are different kinds of knowledge.

(2) Interacting with a player having no knowledge increases knowledge; $k_{i}$ and $k_{j}$ are the same kind of knowledge.

(3) Interacting with a player having full knowledge creates full knowledge; $k_{i}, k_{j}$ are the same kind of knowledge.

The combined knowledge (marked as $k_{(i, j)}$ ) is generated by the following equation:

$$
k_{(i, j)}=\frac{\max \left(k_{i}, k_{j}\right)+\min \left(1, \max \left(k_{i}+k_{j}\right)\right)}{2} .
$$

We define a transferring operator $\oplus$ to describe the knowledge transferring process among players when the game is going. The transferring equation is given below:

$$
K_{i j, t+1}=K_{i, t} \oplus K_{j, t} .
$$

The above method was observed by Szabó and Fáth [5] and Yu et al. [17], with a slightly different definition.

(2) Brand Commitment Model. In marketing, applications of social or group influence can be found across a wide range of contexts [25]. For example, scholars (Arndt [26] and Gatignon and Robertson [27]) have often relied on social influence as a theoretical basis for studying WOM dynamics in the adoption of new products, for understanding reference-group's influence on product and brand choice (Bearden and Etzel [28]) as well as polarization phenomenon in group decision-making (Ward and Reingen [29]). Social identity theory, explained by Ellermers et al. [30] and Tajfel [31], indicates that some people care about the success of the company they identify with. Dutton et al. [32] found that people always voluntarily promote the company on which they have a positive view and become loyal to the brand once they develop to self-identity. Brand commitment is an enduring desire to maintain a relationship with a brand. It can be perceived as a condition in which consumers are firmly enchanted with a certain brand to the extent that there is no second choice. In other words, it implies brand loyalty.

Therefore, if an actor has a high level of commitment to a brand, he or she will tend to keep a stable relationship with the brand. The actions of person with high or low commitment will be different when they receive a negative message talking about a target brand. For the sake of simplicity, we define $b_{i(i=a \text { or } b)}$ as the quantitative measure of brand commitment of the product. This kind of commitment can bring bias when choosing strategy. A value of $b \in[0,1]$ represents player's preference or bias on some brand of product, which means the player will definitely choose certain brand of the product and reject others, no matter which one his neighbours choose. Under some situations we can even infer that they are spokesman or discommender of particular brand. We describe the brand preference level of the two products for player $i$ as a vector $B_{i}=\left(\begin{array}{c}b_{A} \\ b_{B}\end{array}\right)$.

(3) The Rational Choice Model of Conformity. In social commerce, applications of social or group influence can be found across a wide range of contexts; prior results found by Ryu and Han [25] and Arndt [26] suggest that individuals are susceptible to social influence and that they often behave in ways that conform to social norms or pressure. The same conclusion is also observed by Gatignon and Robertson [27] and Bearden and Etzel [28]. Conformity is often meant to represent a solution to the problem and attain or maintain social order that requires cooperation. These studies generally focus on modelling the dynamics of norms in the perspective of cooperation. With reference to the prior research, we assume that the need for social conformity is associated with popularity. Popularity measures player $i$ 's social influence and dominance. It is one of the components in his utility function. For the sake of simplicity, we evaluate it with the number of his neighbours, which is modified from the method suggested by Yu et al. [17]:

$$
P_{i}=\alpha \frac{n_{i}}{n_{\max }} .
$$

In this equation, $n_{\max }$ is the maximum number of neighbours of all players in the network, and $n_{i}$ represents the number of neighbours of player $i . \alpha=[0,1]$ is a controlling 
parameter and describes the probability the player will choose to assimilate. For example, if $\alpha=1$, keeping conformity with his neighbours brings benefit to the player; $\alpha=0$ indicates that the two players' choice conflicts with each other and the total benefit for both two players is 0 . In that case he is more reasonable when making purchase decision.

(4) The Utility Function. Sociologists believe that how human beings interpret and accept things could be summarized to more than one way [33]. The first way is personal experience and findings; another one is the established agreements or beliefs; also some people rely on the behaviours of people in groups, for they insist that believing what others say is a very useful quality that can make people easily get along and contributes a lot to stable social relations. In actual social activities, these three ways are often combined. However giving different weights on them reflects different personalities of people and different characteristics of social behaviour. We believe that the purpose of a rational actor $i$ is to maximize her utility $U_{i}$. Therefore, $U_{i}$ can be defined as a convex combination of information contribution and social norm contribution with coefficients $0 \leq \rho, \pi, k \leq 1$ :

$$
U_{i}=\rho K_{i}+\pi B_{i}+k P_{i} .
$$

With reference to the method and the experienced data recommended by Zinoviev and Duong [8], we use a set of coefficients $\{\rho, \pi, k\}$ to characterize a particular type of actors. For example, $\rho=0, \pi=1$, and $k=0$ describe a type of actors who have high loyalty to a special brand or those who represent the benefit of certain brand. We call them "sworn followers." $\pi=0, \rho=1$, and $k=0$ probably correspond to a community of actors who believe that different self-perceived knowledge level will uniquely determine the strategy-making process; we call them "experts." $\pi=0, \rho=0$, and $k=$ 1 probably correspond to a community of actors who care more about their reputation and conformity with the whole community; we call them "conformists." Meanwhile, $j$ is the neighbour of $i$ in the network. According to the utility definition we can calculate the payoff matrix in detail.

\section{Analysis of Model}

3.1. Assumption of the Model. Product-related information is a kind of public information that can be got from various channels; therefore we modify public goods game model and make the following assumptions:

(1) In social network, the nodes are bounded rational. We agreed that each node only interacts with its neighbour nodes.

(2) Benefit is defined by the payoff matrix. Each node is trying to obtain maximum benefit by enhancing the significance of its activities to improve the social capital in the community.

(3) The evolution process of nodes in the network is influenced by various social factors. Each node has to constantly adjust their strategy, imitating the behaviours of its neighbours to improve the sociability within community.
Strategies in the evolutionary game tend to be influenced by other nodes' opinion in the public information environment. Therefore, the strategy for node $i$ could be described as a dimensional vector $S_{i}$. This kind of game is carried out over certain kinds of social network structures; node $i$ interacts with all of his neighbors in each round, and its payoff $\left(P_{i}\right)$ can be described as

$$
P_{i}=\sum_{j \in \Omega_{i}} s_{i}^{T} M s_{j},
$$

where $\Omega_{i}$ is the set of neighbors of node $i$ in the network and $M$ is the payoff matrix defined in Section 2 .

3.2. Learning and Updating. Each node in the network evolution needs to constantly adjust its strategy to imitate the behaviors of its neighbors to improve sociability, referring to various social factors. Nodes $i$ and $j$ may also change their strategies because they contact public social media or they are influenced by other environment factors. Whether to keep the old strategy or change to the new one is defined by updating rules. At the end of each stage of the evolutionary game, all nodes can adjust their strategy according to the features and benefits of its neighbours. This kind of imitation processes formed a wide class of microscopic update rules. The essence of the imitation is that the node who has the opportunity to revise her strategy takes over one of her neighbor's strategy with some probability. The imitation process covers two respects: whom to imitate and with what probability.

There are several common strategy update rules described in the dynamics of evolutionary games. [5] (Majority rules, Best Response Dynamic, etc.). Among them, this paper considers the typical rule of imitation: Imitate If Better. The standard procedure of the rule is to choose the node to imitate at random from the neighbourhood. In the mean-field case this can be interpreted as a random partner from the whole population. The imitation probability may depend on the information available for the node. The rule can be different if only the strategies used by the neighbours are known, or if both the strategies and their resulting last-round (or accumulated) payoffs are available for inspection. Node $i$ with strategy $S_{i}$ takes over the strategy $S_{j}$ of another node $j$, which is chosen randomly from $i$ 's neighbourhood, if $j$ 's strategy has yielded higher payoff. Otherwise the original strategy $S_{i}$ is maintained. If we denote the set of neighbours of node $i$ who hold the strategy $S_{i}$ by $\Omega_{i}\left(s_{i}\right) \subseteq \Omega_{i}\left(\Omega_{i}(a)+\Omega_{i}(b)=\Omega_{i}\right)$. The individual's strategy transition rate from strategy $s_{i}$ to strategy $s_{i}^{\prime}$ could be written as

$$
\omega\left(s_{i} \longrightarrow s_{i}^{\prime}\right)=\frac{\lambda}{\left|\Omega_{i}\right|} \sum_{j \in \Omega_{i}\left(s_{i}^{\prime}\right)} \theta\left[U_{j}-U_{i}\right],
$$

where $\theta$ is the Heaviside function, $\lambda>0$ is an arbitrary constant, and $\left|\Omega_{i}\right|$ is the number of neighbors. Imitation rules are more realistic if they take into consideration the actual payoff difference between the original and the imitated strategies. Proportional imitation does not allow for an inferior strategy to replace a more successful one. Update rules which forbid this are usually called payoff monotone. 
However, payoff monotonicity is frequently broken in case of bounded rationality. Therefore, a possible general form of imitation more realistic could be described as follows:

$$
P_{r_{i \rightarrow j}}=\frac{1}{1+\exp \left[\left(U_{i}-U_{j}\right) / K\right]},
$$

where $P_{r_{i \rightarrow j}}$ represents the probability node $i$ and imitates the strategy of its neighbour $j$ finally. $U_{j}$ is the benefit generated by node $j$ and it is the maximum among those accrued by its neighbours, while $U_{i}$ is the accumulated benefits of node $i$, and $K$ is the information noise [34], representing the rational level of the nodes.

The smaller $K$ value is, the more rational of the behaviour is. If $K=0$, then the whole process can be described as a completely rational game, in which there is no error when making decision; $K=+\infty$ indicates that game strategies choosing can be depicted as a pure random process, which is completely irrational; $0<K<+\infty$ indicates a limited rational game process; namely, the gamers adjust their strategies according to rules, but there is a certain error.

\section{Computational Simulations}

In this section, we design the computational system, simulate the virtual community and evolutionary process, collect numerical results under different scenarios, and make comparisons through statistical analysis. The simulation and experiments are implemented by NetworksX 1.7. NetworkX is a graph theory and complex network modelling tool, developed with Python language, containing built-in algorithms figure and complex network analysis modules, which can be conveniently imported and executed for complex network data analysis, simulation modelling, and so forth.

4.1. Formalize the Model Definition. Let each node in the virtual network be an agent and there are $n$ agents. We define the community network $N$ as $N=\{X, \mathrm{NT}, U, F, t\}$, in which

(1) $X$ is the set of agents and $X=$ agent1, agent2, $\ldots$, agent $n$. Each agent is a node in the network.

(2) NT is the set of network types and NT = \{smallword, random, scalefree\}.

(3) $U$ is the set of benefits derived by all agents in each game round; it is the accumulated value while gaming with all its neighbours, $U=\left\{U_{1}, U_{2}, \ldots, U_{n}\right\}$.

(4) $F$ is the state transfer function. The state of agent $i$ at time $t+1$ is a function of parameters as stated in (12).

4.2. Experimental System and the Default Parameters. In our study, a node in the network is either in a cooperation (strategy $a$ ) or defection (strategy $b$ ) state. We describe the diffusion of information for product $A$ (information $A$ for simplicity in the later paper) in the network as

$$
\begin{aligned}
& \text { diffusion of information } A(\%) \\
& =\frac{\text { sum of the nodes holding strategy } a}{\text { total number of nodes }} * 100 \% \text {. }
\end{aligned}
$$

And we describe the diffusion of information for product $B$ (information $B$ for simplicity) as

$$
\begin{aligned}
& \text { diffusion of information } B(\%) \\
& =\frac{\text { sum of the nodes holding strategy } b}{\text { total number of nodes }} * 100 \% \text {. }
\end{aligned}
$$

In the model, we simulate individual strategy evolution. The evolutions of the information spreading and accepting conditions are studied, trying to find out how to promote or hinder certain preference of strategy from winning. We conduct several experiments on the competitive information dissemination in a social network. There are some familiar and natural models of social networks, which could be applied in these experiments: random graph, scale-free network, and small-world network. We applied the latter two different networks for case study. A scale-free network is a random graph whose degree distribution follows a power law, while a small-world network follows a random graph model in which most nodes can be reached from every other node by a small number of hops, which is generally known as smallworld phenomenon. Many empirical networks are wellmodelled by small-world networks, such as social networks, wikis, and gene networks.

We explore the interaction among all individuals over time (100 simulation rounds in our case). The simulation is divided into five procedures.

(1) Set up the network structure; define the game parameters and the model parameters.

(2) Graphically illustrate the network evolution and show how the nodes change dynamically in the network.

(3) In each step, each participating individual updates the strategy according to the rules of replication dynamic view (strategy).

(4) In each simulation time period, take the evolutionary steps in the periodic system that all the individuals or a fixed percentage of them reach or maintain a stable state as the equilibrium of the evolution.

(5) Display the simulation result and show the characteristics of the network.

4.3. Experimental Results. The population of our experiments is set to 1000. The small-world network applied for our experiments is set as follows: the number of connections per node is set to 5 , and the probability of connection to link neighbours per node is set to 0.2 . For the scale-free network, the number of edges being added to the network each step is set as $5 . k_{a}, k_{b}, b_{a}$, and $b_{b}$ are initialized using uniform distribution on $[0,1)$ throughout the experiment with slight difference under different scenarios.

(1) Results for Different Network Structures. At the start of the experiments, the two pieces of product information has been equally adopted. $k_{a}, k_{b}, b_{a}$, and $b_{b}$ are initialized using uniform distribution, with the condition that $A$ dominate in dissemination during gaming. We assume that the maximum 


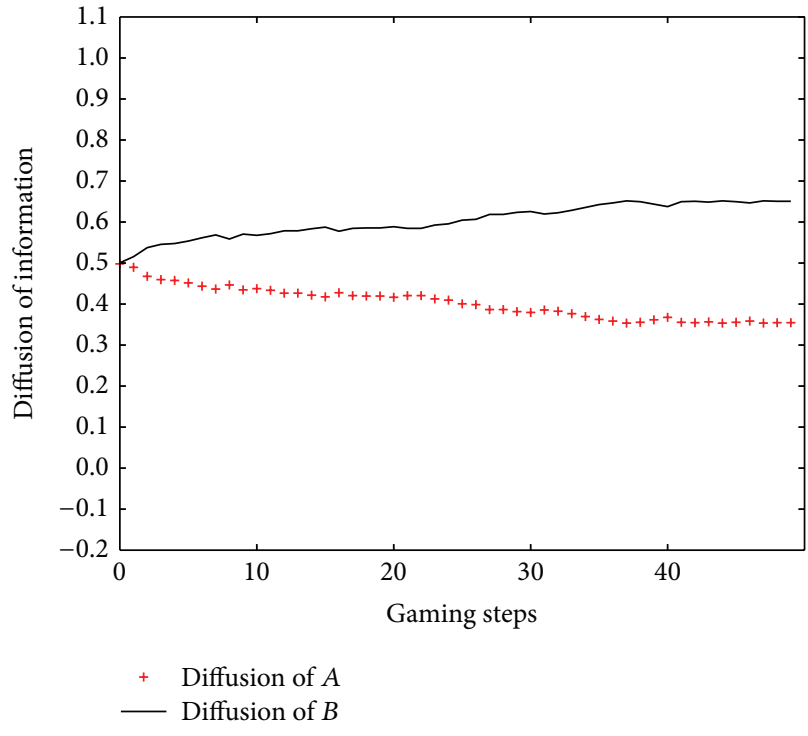

(a) Information diffusions in small-world networks

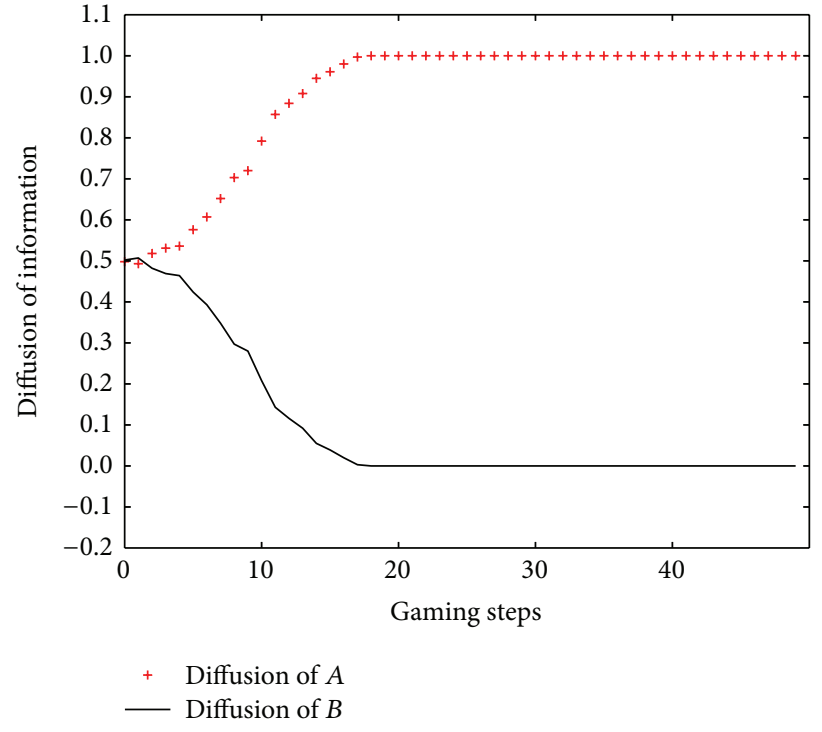

(b) Information diffusions in scale-free networks

FIGURE 1: \% of adoption for competitive product-related information under different network structure.

value of $k_{a}$ is larger than the one of $k_{b}$; for simplicity, to guarantee the condition, $k_{a}$ and $k_{b}$ are initialized on $[0.2,0.7]$ and $[0,0.2)$ and vary following the knowledge dynamic rules defined in Section 2. $b_{a}$ and $b_{b}$ follow uniform distribution on the interval $[0,1) . \pi=0.3, \rho=0.3, k=0.4$, and $K=0.8$. The evolutionary steps in each round are set to 50 .

Following the conditions of this scenario, we find the dominant strategy reaches the whole population or reaches a stable dominant winning status (the equilibrium), in different gaming steps over different network structure (see Figure 1). In the scale-free network, the dynamic can reach a stable status more easily.

Compared to the small-world networks, in scale-free network, the majority of individuals have only one highdegree neighbour. There will be more references for him to evaluate his neighbours' payoff, providing him with a higher probability to successfully imitate a better strategy. Then the neighbours of him are apt to imitate his strategy because of his high-degree status in the social networks. After several game rounds, the individual game is changed to group game with several high-degree players as the core nodes. It is obvious that the number of groups is much less than the number of nodes who participate in the game; thus the Scalefree networks can reach equilibrium faster than small-world model and meanwhile shows higher sensitivity, assumed by a larger amplitude around the equilibrium.

(2) Results for Different Personalities. Our analysis continues with how the spreading of competitive information differs according to the weight of acceptance change that affects agents' utility function. In the prior model, we classify the cases into different personalities.

(a) The Influence of Individual Personality Differences on Game Equilibrium. We use a particular set of coefficients $\{\rho, \pi, k\}$ to characterize different types of agents. At the start of experiments, $k_{A}, k_{B}, b_{A}$, and $b_{B}$ are initialized using uniform distribution on the internal $[0,1)$ and they evolve according to the knowledge dynamic rules described in Section 2. Referring to the empirical data [17] (Yu et al., 2012), $K=$ 0.01 . We ran the experiment in three different cases which cover population with different personalities. In the first case (Figure 2), the agents have high desire for knowledge and low desire for brand loyalty and reputation; we set the parameters $(\pi, \rho, k)$ distinguishing personalities as $(0.8,0.1,0.1)$, which we called "experts." In the second case, the agents have low expectation for reputation and knowledge and strong desire for loyalty to a certain brand; we set the parameter $(\pi, \rho, k)$ as $(0.1,0.8,0.1)$, and we called them "sworn followers." In the last case, the agents have great desire for social conformity and influence; we set the parameter $(\pi, \rho, k)$ as $(0.1,0.1,0.8)$ and call them "conformist."

In this experiment, the gaming steps are set to 50 in each simulation round and the initial \% of information $A$ is set to $80 \%$ to get a more definite comparison. We carry out experiments in 100 simulating rounds totally for the two different kinds of network structure.

Comparing experimental results under three different scenarios, a phenomenon could be observed in common, that the average steps before reaching the equilibrium in scale-free network is generally less than that in the smallworld network, which get the consistent conclusion with experiment (a). The convergence of dynamic in scale-free network is more obvious with slight fluctuation, while the dissemination in the small-world network distribute more scattered.

As one can see from the figures, in all scenarios the whole network rapidly converges to a stable distribution. In the network of "experts" the average full convergence takes more time than in the network of "sworn followers" and 


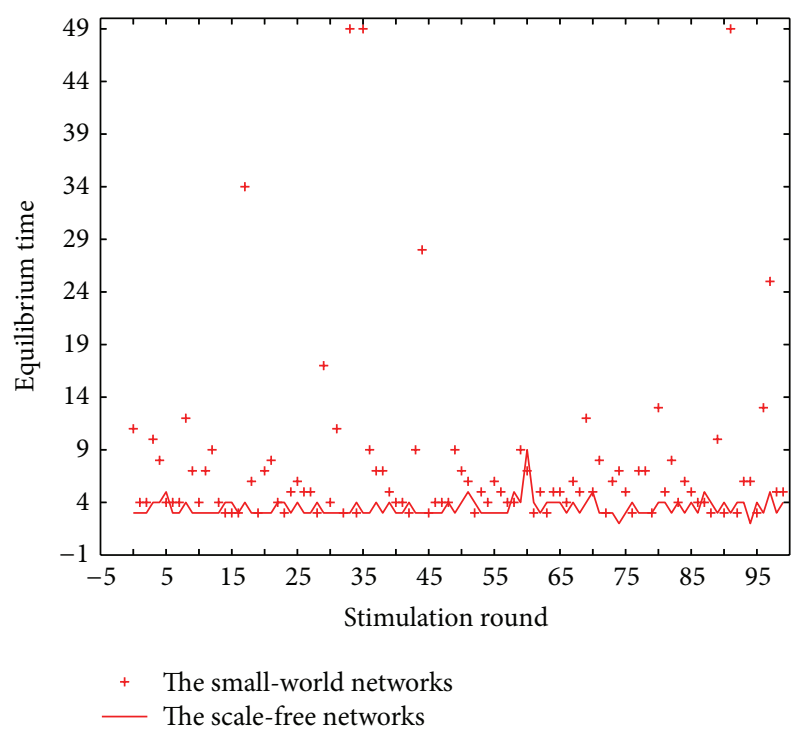

(a) Information diffusions among "Experts"

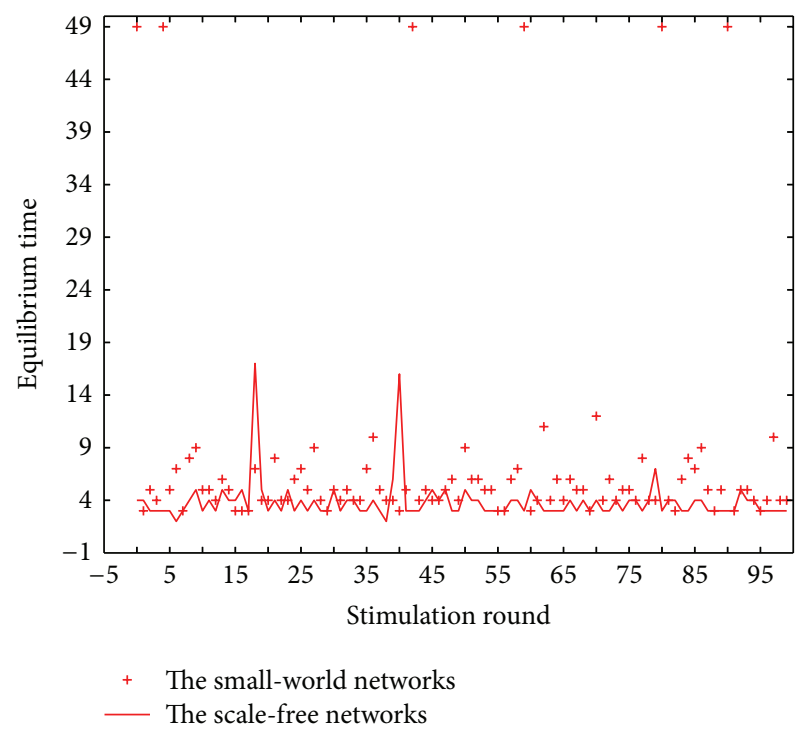

(b) Information diffusions among "Sworn followers"

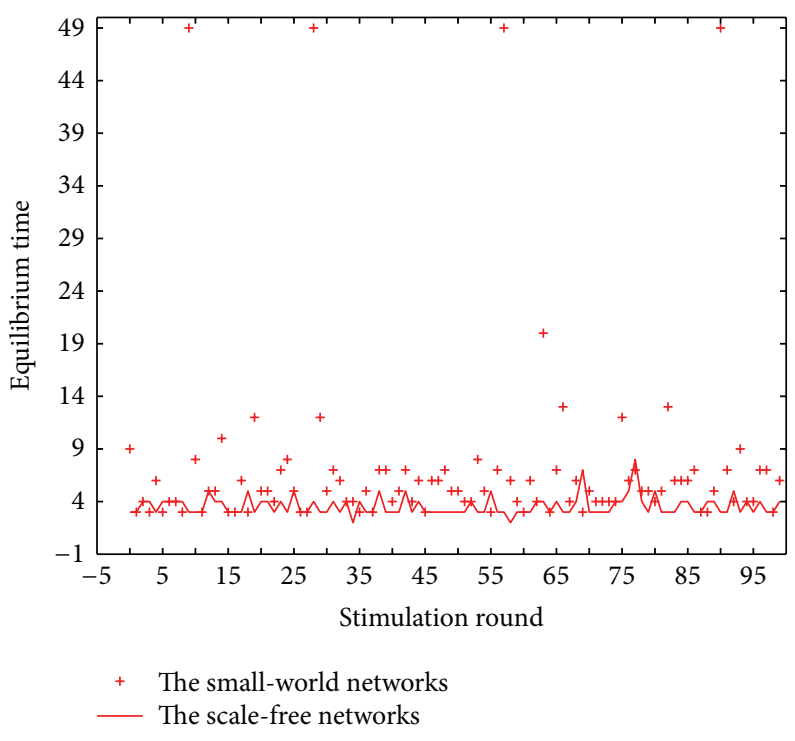

(c) Information diffusions among "Conformists"

FIGURE 2: Different convergence times due to different settings of $(\pi, \rho, k), k_{A}, k_{B}, b_{A}$, and $b_{B}$ are initialized on $[0,1)$. The values of $(\pi, \rho, k)$ are, from (a) to (c), $(0.8,0.1,0.1),(0.1,0.8,0.1)$, and $(0.1,0.1,0.1)$, representing experts, sworn followers, and conformists.

"conformist." Also, the "expert" network always has some unsubstantial numbers of people doubting the diffused facts, performance in the sharp fluctuations. Generally speaking, information dissemination will proceed to the stable state most quickly in the scale-free network with "conformist" in the majority.

Local heterogeneity behaviour preference can explain the phenomenon, which could be sharply reduced over the network of "sworn followers" and "conformist." A compatible opinion or preference could be more easily reached, being explained by fewer steps before the equilibrium during evolution. Each agent considers all the strategies their neighbours take and keep consistent with the overall opinion easily. However, when "experts" take a strategy, accidental shadows have more chance to happen, which increase the uncertainty of spreading result.

(b) The Influence of Preference Benefit on Information Dissemination. Large proportion of a particular personality dominant in the population indicates a certain psychological tendency in social networks. For example, the psychology of "First Impression" leads to a majority of "sworn followers" to a special brand or product. The psychology of "Herd effect" leads to plenty of "conformists." Meanwhile, the psychology of "Experientialism" makes most agents in the network more rational and specialistic.

In addition, a piece of information has its own life cycle, experiencing the process of production, diffusion, decay, and 

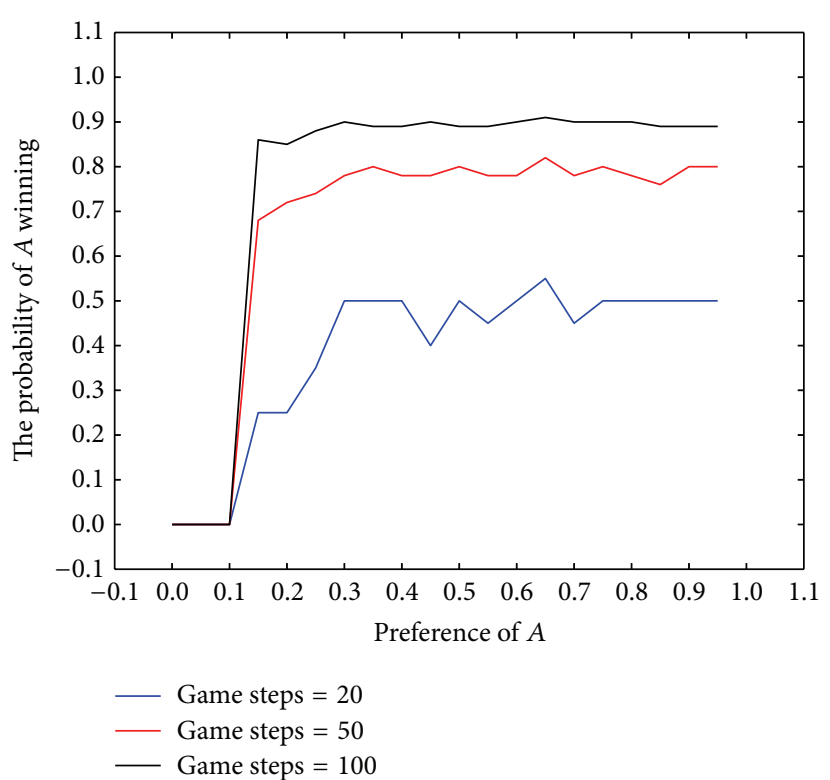

(a) Probability for winning of information $A$ with yield change of $A$ 's preference benefit

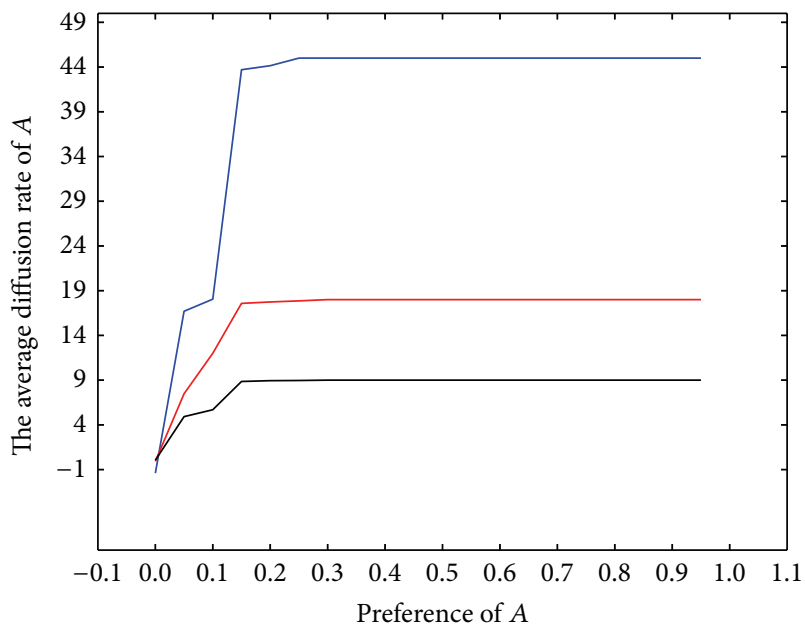

$\begin{aligned}- \text { Game steps } & =20 \\ - \text { Game steps } & =50 \\ \text { Game steps } & =100\end{aligned}$

(b) Average diffusion rate of information $A$ with yield change of $A$ 's preference benefit

Figure 3: Under different evolutionary time, the diffusion states of information $A$ with yield change of $A$ 's preference benefit (relative to $B$ ). The values of $b_{A}$ and $b_{B}$ are well-distributed on $[0,1)$ with step size $0.05 . k_{A}, k_{B}$ are initialized using uniform distribution on the internal $[0,1)$.

death, which cannot be absolutely fixed. Therefore, each round of evolutionary game cannot continue without time limiting. Thus, we simulate the winning probability of certain information for evolutionary process in limited time periods, trying to reveal the dissemination of competitive information from a more practical perspective.

As is shown in Figure 3, the gaming steps are, the gaming steps are, respectively, set to 20,50, and 100; the initial \% of information $A$ is set to relatively low rate as $1 \%$. Taking the small-world network under the psychological state of "First Impression" for instance, we carry out experiments in 100 rounds for different evolution time, trying to observe the change on winning probability and diffusion rate of information $A$ due to different information preference benefits.

The winning probability of $A$ generally increases with its preference benefit and the evolution time. When the benefit is lower than 0.1 comparing to $B$, there is no advantage for $A$ to win. There is a sharp rising between the preference values 0.1 and 0.2 . After that, the probability fluctuates at a relatively high level. Following the rule of "Imitate If Better," a very small group of initial adoption can get a high win probability. The diffusion rate of $A$ also shows an ascending trend along with the preference benefit increasing when the benefit is lower than 0.2. Then the average diffusion rate keeps stable. Meanwhile, it can be concluded that information diffuse becomes lower after 50 game steps, which means, in most rounds of simulation, the minority of population for information $A$ will quickly occupy the whole population because of the enough preference benefit and knowledge dynamic.

(3) Results with Different Initial Settings. We now discuss the impact of the initial \% of information settings. The initial percentage of people adopting information $A$ is set at $0.1,0.5$, and 0.8 , respectively. $k_{a}$ and $k_{b}$ are initialized on [0.2,0.7] and $[0,0.2)$, varied by the knowledge dynamic rules defined in Section 2. $b_{a}$ and $b_{b}$ follow uniform distribution on the interval $[0,1) . \pi=0.3, \rho=0.3, k=0.4$, and $K=0.8$. The evolution steps in each round are set to 50 . The simulation round is set to 100 and the value gained at each step is calculated to the average.

The results indicate that the initial support of information will also have a major impact on the diffusion process. If information $A$ in the initial state of the system has a higher adoptive rating $(p>0.5)$, the chance of information $A$ wins will be further enhanced. As to the lower initial adoptive rating of information $A(p<0.5)$, the network will take much more time to reach an equilibrium as shown in Figure 4 . In the network structure of small-world, information $A$ will fail to win when its initial adoptive rating is set to 0.1 . When information $A$ 's initial adoptive rating is increasing, the diffusion rate of $A$ raises sharply, indicating that information $A$ is more likely to reach the whole population. Under the structure of scale-free network, the phase change process is much smoother. It can be inferred that scale-free networks provide more heterogeneous topology structure, in which information $A$ has a higher probability of winning. Thus scale-free networks could be seen as a factor which decreases with the winning odds of preferred information for a certain product.

(4) Results with Incomplete Information. We finally examine the impact of information noise on the probability of $A$ 's adoption and dissemination. The information noise levels are set at $k=0.01,10$, and 10,000 , respectively. 


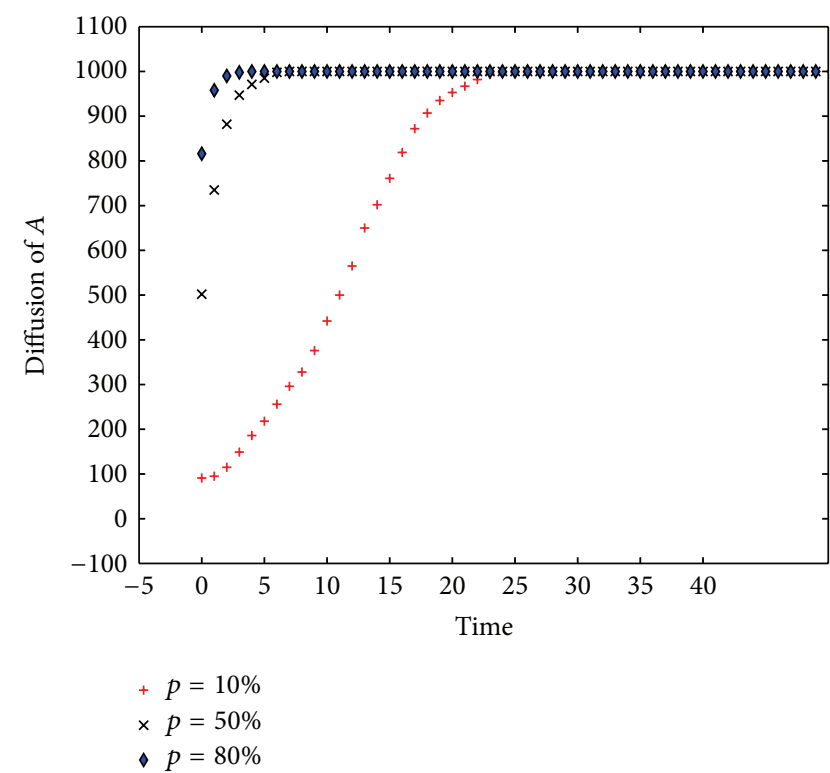

(a) Diffusion of information $A$ under different initial \% setting in smallworld networks

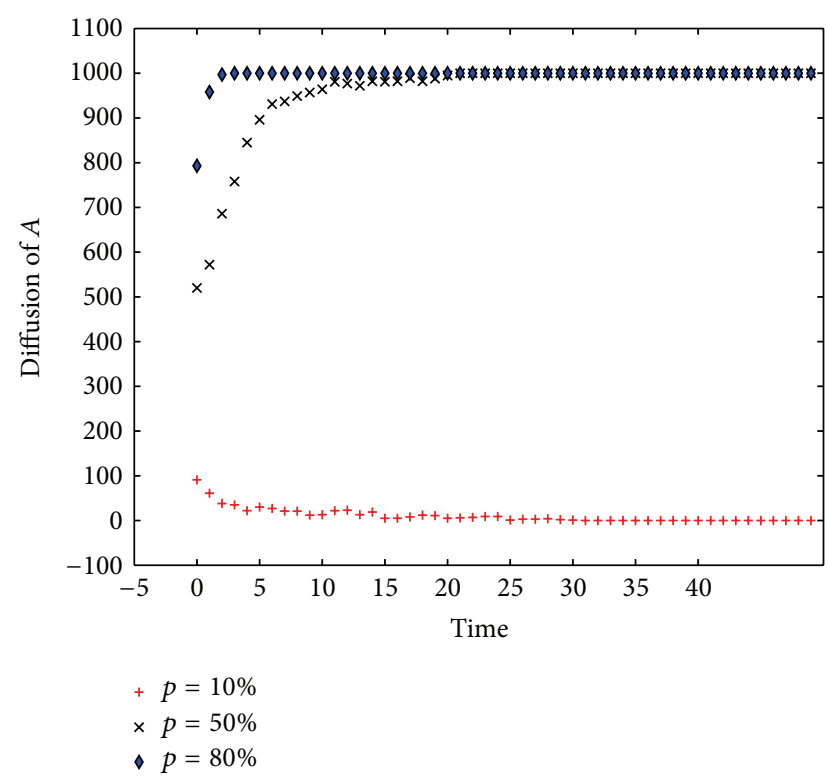

(b) Diffusion of information $A$ under different initial \% setting in scalefree networks

FIGURE 4: Diffusion of information under different initial \% setting through different network structure. The value of $p$ is $0.1,0.5$, and 0.8 .

The initial percentage of people adopting information $A$ is set at 0.1 to make a more significant comparison. $k_{a}$ and $k_{b}$ are initialized on $[0.2,0.7]$ and $[0,0.2)$ and vary following the knowledge dynamic rules defined in Section 2. $b_{a}$ and $b_{b}$ follow uniform distribution on the interval $[0,1) . \pi=0.3$, $\rho=0.3$, and $k=0.4$. The evolution steps in each simulation time period are set to 50 . The simulation round is set to 100 and in each simulation period the mean value is referred.

The results in Figure 5 reveal that individual's irrational selection of strategies will also affect the results of dissemination. The smaller $K$ 's value is, the more rational behaviour can be observed. The preferred information will take the advantage more easily over the network. It indicates that, for a limited rational game process, namely the game players adjust their strategies according to certain rules, the evolution will reach an equilibrium under the circumstance of low knowledge noise. Therefore, in the process of irrational game, it is hard for a certain piece of information to win more audience than the other, even though the former one has an obvious advantage of initial share. The reason is that the increasing of noise brings uncertainty of strategy evolution when "Imitate If Better" updating rule plays a role. It can also easily reach the conclusion again that scale-free networks structure could weaken the winning probability of preferred information.

\section{Conclusion and Future Work}

An evolutionary game model based on stochastic strategy updating dynamic for competitive product-related information dissemination in social network is presented in this paper. Several important implications can be drawn from the work.
Firstly, compared with the Small-world networks, Scalefree networks are a kind of better structure for information dissemination. In the case that the overall average knowledge level is relatively high, the advantageous information in scalefree networks more easily defeat the others because of the less difference between individuals in this network structure.

Secondly, different virtual communities (with different network structures) should adopt different macrolevel managerial strategies, for instance customized rewarding and penalty mechanisms. Different incentives and penalties can influence user's choice of product-related information spreading decision and affect the performance of information sharing.

Furthermore, results of the competitive information dissemination are affected by the initial self-perceived knowledge distribution of products and brand preference among individuals, which means that, in order to make more individuals prefer a brand than the other, the company should provide more information to indirectly promote individuals' acceptance of the product, for example, through the mass media.

Finally, different personalities and mentalities in the virtual community lead to different dissemination process of competitive product-related information. Individuals' irrational selection of strategies also affects the results of dissemination.

Other models as we know, including SIR Model, Markov Model, and Random Petri-Net Model, only explain the information spreading in individual behaviour level. They could not preferably reflect the individual spreading behavior evolution in the context of time change, network structure, and interaction with others. Game theory is powerful tool in information dissemination behavior description. Our work 


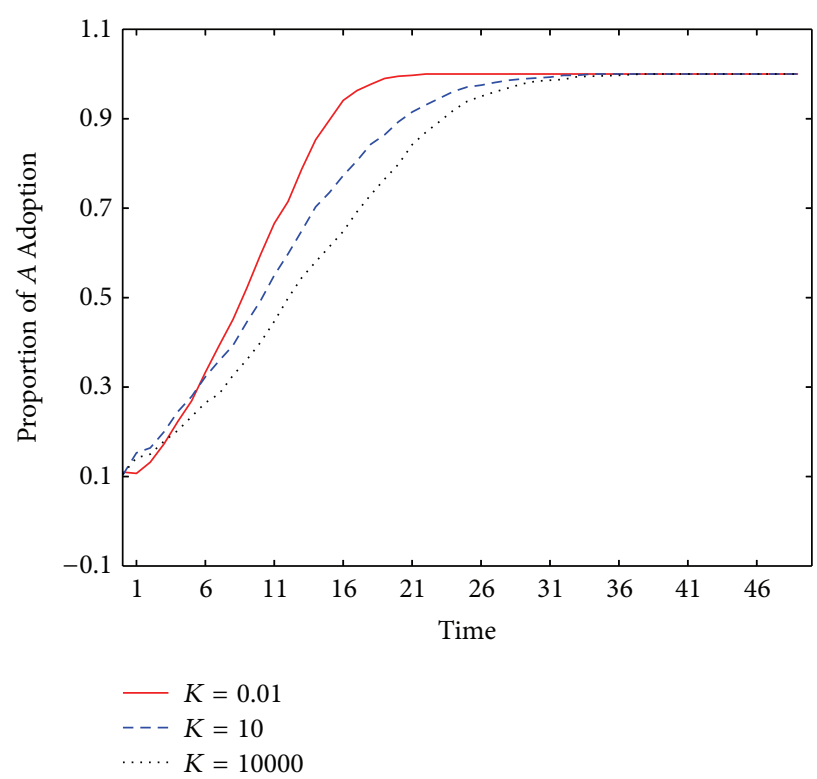

(a) The trend of change for information $A$ adoption in evolution over small-world networks

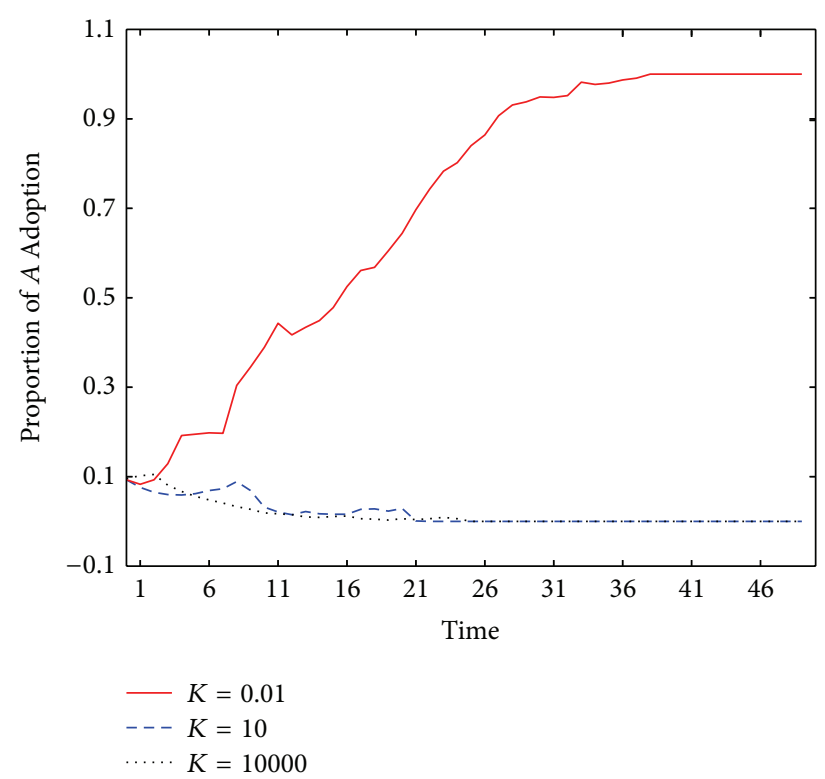

(b) The trend of change for information $A$ adoption in evolution over scale-free networks

FIGURE 5: The impact of information noise on the $\%$ of information adoption in evolutionary process, the value of $K$ is $0.01,10$, and 1000 .

reveals several interesting conclusions as to the nature of competitive information dissemination, employing an agentbased simulation method to explore the evolution of information adoption and dissemination behavior in social networks. We hope that this paper would provide some new insights into the research of competitive information.

This paper has some limitations and we will improve our model from the aspects as follows.

(1) We assume that the network structure is fixed and did not consider the evolution of the network structures themselves, including not only homogeneous network, but also heterogeneous network containing several different types of players.

(2) The utility parameters are endogenous to some extent. To evaluate how the endogeneity impacts the personality and to what extent it will influence the choice of information adoption is a problem we will discuss in the future work.

(3) Large number of real data should be used to support the model, as a supplement of the simulation.

\section{Conflict of Interests}

The authors declare that there is no conflict of interests regarding the publication of this paper.

\section{Acknowledgment}

This work is supported by the National Natural Science Foundation of China (NSFC: 71271012 and NSFC: 71332003).

\section{References}

[1] A. Montanaria and A. Saberi, "The spread of innovations in social networks," Proceedings of the National Academy of Sciences of the United States of America, vol. 107, no. 47, pp. 20196-20201, 2010.

[2] D. Gruhl, R. Guha, D. L. Nowell, and A. Tomkins, "Information diffusion through blogspace," in Proceedings of the 13th International Conference on World Wide Web (WWW'04), pp. 491-501, 2004.

[3] J. Yang and J. Leskovec, "Modeling information diffusion in implicit networks," in Proceedings of the 10th IEEE International Conference on Data Mining (ICDM '10), pp. 599-608, IEEE, Sydney, Australia, December 2010.

[4] L. Lü, D.-B. Chen, and T. Zhou, "The small world yields the most effective information spreading," New Journal of Physics, vol. 13, Article ID 123005, 10 pages, 2011.

[5] G. Szabó and G. Fáth, "Evolutionary games on graphs," Physics Reports A, vol. 446, no. 4-6, pp. 97-216, 2007.

[6] J. Kostka, Y. A. Oswald, and R. Wattenhofer, "Word of mouth: rumor dissemination in social networks," in Structural Information and Communication Complexity, vol. 5058 of Lecture Notes in Computer Science, pp. 185-196, Springer, Berlin, Germany, 2008.

[7] D. Meier, Y. A. Oswald, S. Schmid, and R. Wattenhofer, "On the windfall of friendship: inoculation strategies on social networks," in Proceedings of the 9th ACM Conference on Electronic Commerce (EC '08), pp. 294-301, July 2008.

[8] D. Zinoviev and V. Duong, "A game theoretical approach to broadcast information diffusion in social networks," in Proceedings of the 44th Annual Simulation Symposium, pp. 4752, 2011.

[9] D. Zinoviev, V. Duong, and H. G. Zhang, "A game theoretical approach to modeling information dissemination in social networks," in Proceedings of the SUMMER 4th International 
Conference on Knowledge Generation, Communication and Management, vol. 1, pp. 407-412, 2010.

[10] G. Jiang, F. Ma, J. Shang, and P. Y. Chau, "Evolution of knowledge sharing behavior in social commerce: an agentbased computational approach," Information Sciences, vol. 278, pp. 250-266, 2014.

[11] N. Alon, M. Feldman, A. D. Procaccia, and M. Tennenholtz, "A note on competitive diffusion through social networks," Information Processing Letters, vol. 110, no. 6, pp. 221-225, 2010.

[12] Y. Wang, J. Li, K. Meng, C. Lin, and X. Cheng, "Modeling and security analysis of enterprise network using attack-defense stochastic game Petri nets," Security and Communication Networks, vol. 6, no. 1, pp. 89-99, 2013.

[13] R. Takehara, M. Hachimori, and M. Shigeno, "A comment on pure-strategy Nash equilibria in competitive diffusion games," Information Processing Letters, vol. 112, no. 3, pp. 59-60, 2012.

[14] J. R. Davis, Z. Goldman, E. N. Koch et al., "Equilibria and efficiency loss in games on networks," Internet Mathematics, vol. 7, no. 3, pp. 178-205, 2011.

[15] A. Galeotti, S. Goyal, M. O. Jackson, F. Vega-Redondo, and L. Yariv, "Network games," The Review of Economic Studies, vol. 77, no. 1, pp. 218-244, 2010.

[16] G. Jiang, B. Hu, and Y. Wang, "Agent-based simulation of competitive and collaborative mechanisms for mobile service chains," Information Sciences, vol. 180, no. 2, pp. 225-240, 2010.

[17] J. Yu, Y. Wang, J. Li, H. Shen, and X. Cheng, "Analysis of competitive information dissemination in social network based on evolutionary game model," in Proceedings of the 2nd International Conference on Cloud and Green Computing (CGC '12), pp. 748-753, Xiangtan, China, November 2012.

[18] J. Hofbauer and K. Sigmund, "Evolutionary game dynamics," Bulletin of the American Mathematical Society, vol. 40, no. 4, pp. 479-519, 2003.

[19] S. E. Asch, “Opinions and social pressure," Scientific American, vol. 193, no. 5, pp. 31-35, 1955.

[20] S. Schachter, "Deviation, rejection, and communication," Journal of Abnormal and Social Psychology, vol. 46, no. 2, pp. 190207, 1951.

[21] M. Deutsch and H. B. Gerard, "A study of normative and informational social influences upon individual judgment," Journal of Abnormal and Social Psychology, vol. 51, no. 3, pp. 629-636, 1955.

[22] D. McQuail, Mass Communication Theory: An Introduction, Sage Publications, Thousand Oaks, Calif, USA, 2nd edition, 1987.

[23] A. J. Flanagin and M. J. Metzger, "Internet use in the contemporary media environment," Human Communication Research, vol. 27, no. 1, pp. 153-181, 2001.

[24] L. F. Feick and L. L. Price, "The market maven: a diffuser of marketplace information," Journal of Marketing, vol. 51, pp. 8397, 1987.

[25] G. Ryu and J. K. Han, "Word-of-mouth transmission in settings with multiple opinions: the impact of other opinions on WOM likelihood and valence," Journal of Consumer Psychology, vol. 19, no. 3, pp. 403-415, 2009.

[26] J. Arndt, "Role of product-related conversations in the diffusion of a new product," Journal of Marketing Research, vol. 4, no. 3, pp. 291-295, 1967.

[27] H. Gatignon and T. S. Robertson, "A propositional inventory for diffusion research," Journal of Consumer Research, vol. 11, pp. 849-867, 1985.
[28] W. O. Bearden and M. J. Etzel, "Reference group influences on product and brand purchase decisions," Journal of Consumer Research, vol. 9, no. 2, pp. 183-194, 1982.

[29] J. C. Ward and P. H. Reingen, "Sociocognitive analysis of group decision making among consumers," Journal of Consumer Research, vol. 17, pp. 245-265, 1990.

[30] N. Ellermers, R. Spears, and B. Doosje, Social Identity, Blackwell, Oxford, UK, 1999.

[31] H. E. Tajfel, Differentiation between Social Groups: Studies in the Social Psychology of Intergroup Relations, Academic Press, Oxford, UK, 1978.

[32] J. E. Dutton, J. M. Dukerich, and C. V. Harquail, “Organizational images and member identification," Administrative Science Quarterly, vol. 39, no. 2, pp. 239-263, 1994.

[33] E. R. Babbie, The Practice of Social Research, Wadsworth Publishing Co Inc., 11th edition, 2007.

[34] W.-B. Du, X.-B. Cao, M.-B. Hu, H.-X. Yang, and H. Zhou, "Effects of expectation and noise on evolutionary games," Physica A: Statistical Mechanics and its Applications, vol. 388, no. 11, pp. 2215-2220, 2009. 


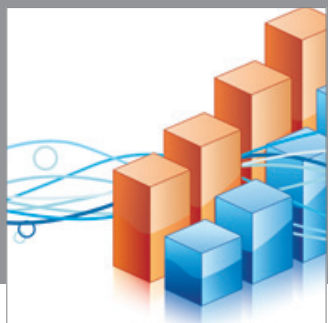

Advances in

Operations Research

mansans

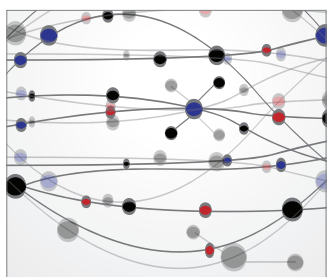

The Scientific World Journal
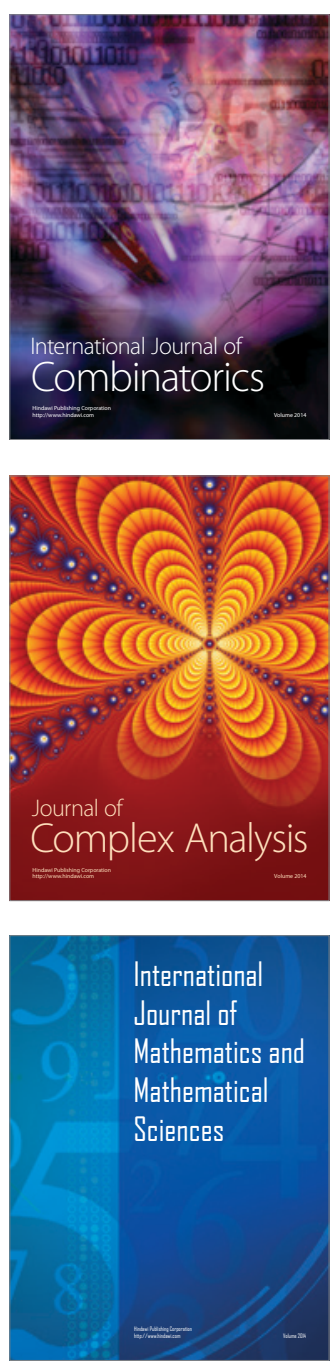
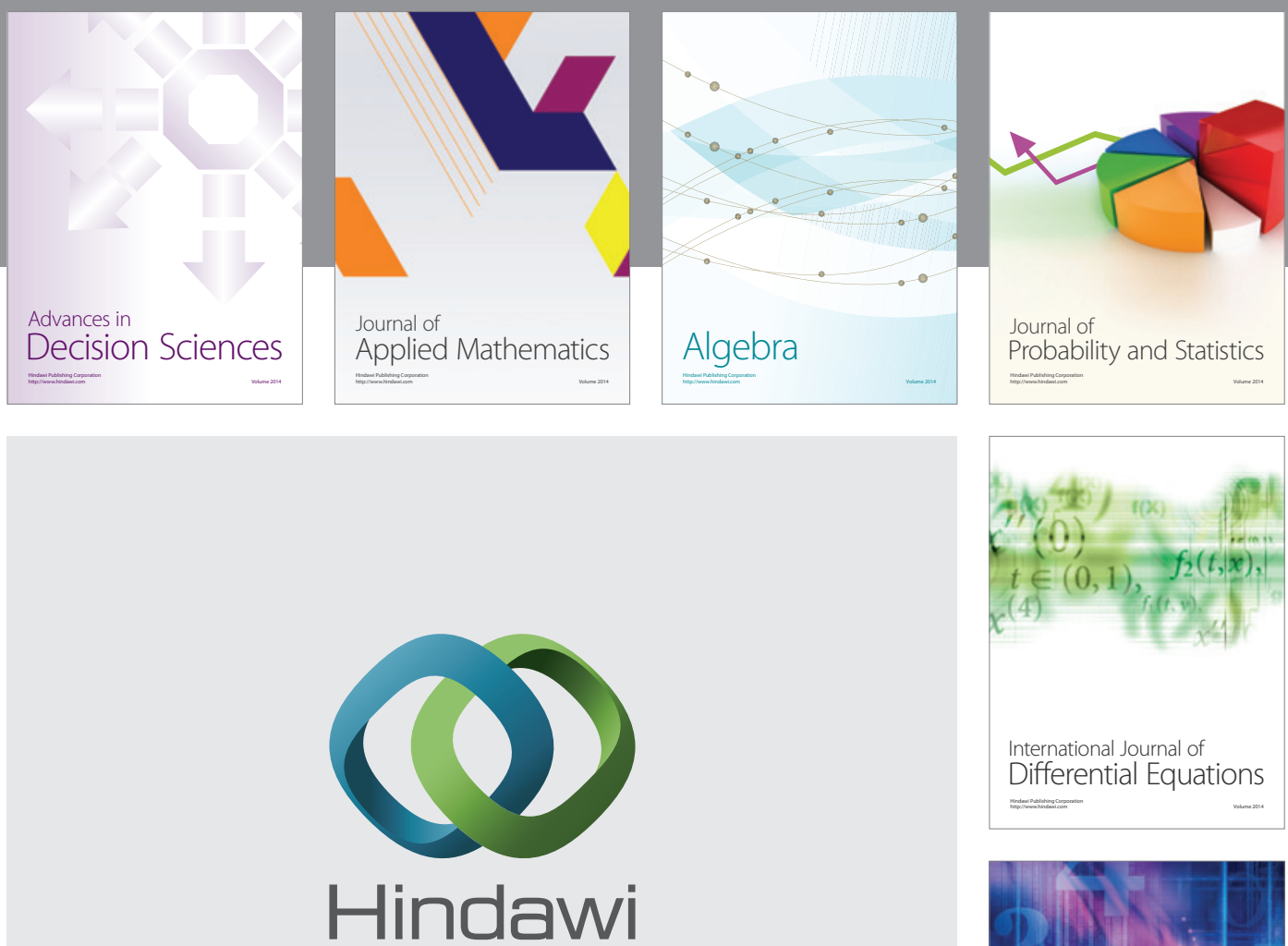

Submit your manuscripts at http://www.hindawi.com
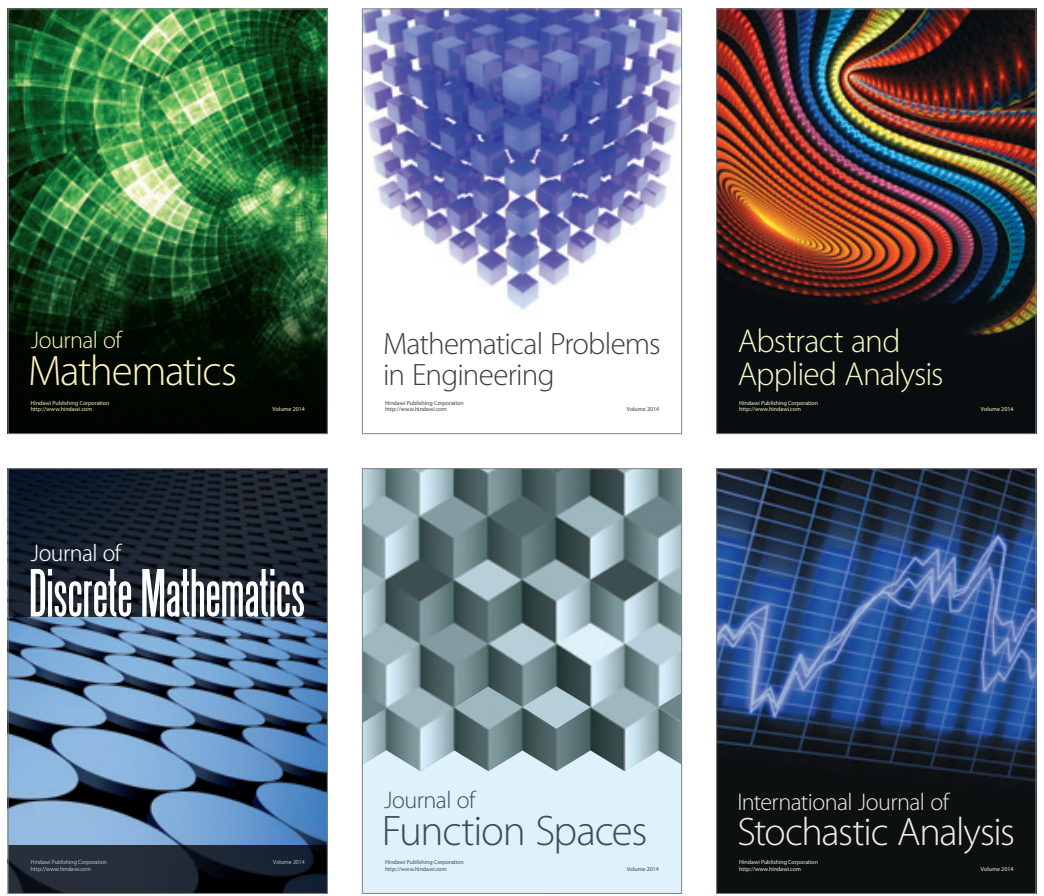

Journal of

Function Spaces

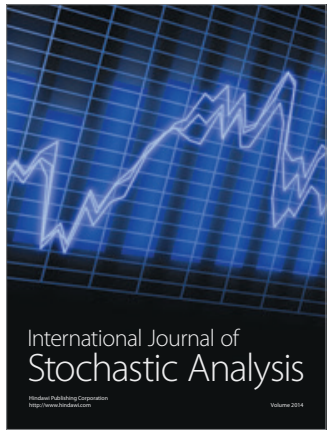

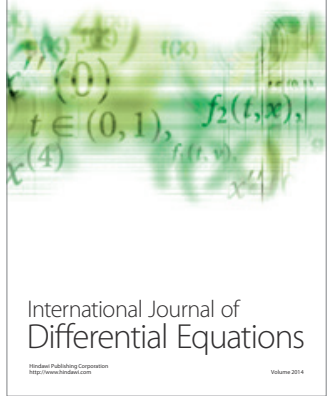
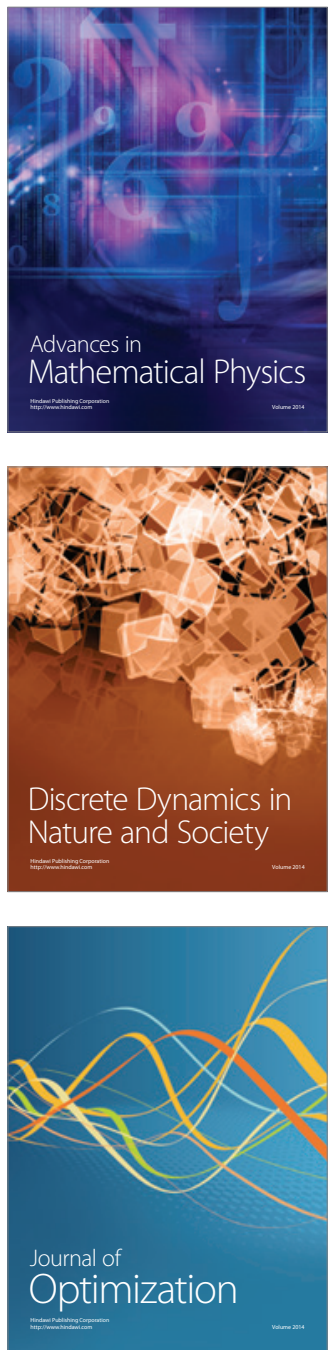